\title{
Approche méthodologique pour la validation environnementale d'un projet de remblaiement de cavités terrestres de la zone littorale à l'aide de sédiments de dragage de ports maritimes traités
}

\author{
Yves PERRODIN $^{1}$, Robert MORETTO $^{2}$
}

1. Université de Lyon, ENTPE, Laboratoire LEHNA, UMR CNRS 5023, 2 rue Maurice Audin,69518 Vaulx-en-Velin Cedex, France. yves.perrodin@entpe.fr

2. Insavalor SA, GIS EEDEMS, 66 boulevard Niels Bohr, BP 52132, 69603 Villeurbanne Cedex, France.robert.moretto@insavalor.fr

\section{Résumé :}

Les sédiments de dragage des ports maritimes non rejetables en mer en raison de leur forte pollution posent un problème de gestion dans la mesure où les filières terrestres classiques (incinération, mise en décharge,...) ne sont adaptées à leur traitement, ni sur le plan économique, ni sur le plan des volumes absorbables. Parmi les solutions de substitution envisagées, le remblaiement de carrières sèches représente une filière prometteuse, notamment parce qu'elle offre des capacités suffisantes pour gérer de tels volumes. Cette filière nécessitait toutefois d'engager un programme de recherche visant à proposer une méthodologie d'évaluation des risques écologiques spécifique, permettant de valider, lors de chaque opération de remblaiement d'envergure, sa compatibilité avec les milieux et les écosystèmes continentaux voisins.

Dans ce contexte, l'objectif du programme ANR SEDIGEST était double :

- sur le plan opérationnel : développer une méthodologie d'évaluation des risques écologiques adaptée au scénario de gestion précédemment évoqué,

- sur le plan scientifique : lever les principaux verrous qui s’opposaient à la rédaction d'une telle méthodologie, et qui étaient, pour l'essentiel, liés à une compréhension encore très partielle des différents mécanismes physiques, chimiques et biologiques qui interviennent lors de leur dépôt à terre.

Après trois années de travaux, portant sur trois matrices sédimentaires originaires de ports maritimes du sud et de l'ouest de la France (deux du Var et un du Finistère), le programme SEDIGEST a abouti à une proposition méthodologique, comprenant quatre niveaux de complexité possibles, utilisable pour la validation environnementale d'un projet donné de remblaiement de cavités terrestres à l'aide de sédiments de dragage portuaires.

Soumis le 6 janvier 2012, accepté le 27 février 2012, en ligne le 16 avril 2012.

La seule version examinée est celle écrite en français. La ou les autres versions n'étant pas examinées par le comité de rédaction de la revue, sont donc publiées sous l'entière responsabilité du ou des auteurs.

A TRANSLATED VERSION IN ENGLISH IS AVAILABLE ONLINE

Pour citer cet article :

PERRODIN Y., MORETTO R. (2012). Approche méthodologique pour la validation environnementale d'un projet de remblaiement de cavités terrestres de la zone littorale à l'aide de sédiments de dragage de ports maritimes traités. Revue Paralia, Vol. 5, pp 2.1-2.12.

DOI:10.5150/revue-paralia.2012.002 ～(disponible en ligne - http:/www.paralia.fr - available online) 


\section{Introduction}

Depuis une vingtaine d'années, sur le plan international, les sédiments de dragage des ports maritimes font l'objet de procédures de gestion de plus en plus élaborées en raison de leur pollution liée aux activités industrielles, commerciales, urbaines et touristiques qui s’y déroulent (LAU et al., 1993 ; ANDERSEN et al., 1998 ; MEEDDAT, 2008). Les principales familles de polluants concernés sont les métaux lourds (ROMANO et al., 2004 ; COLACICCO et al., 2010 ; LEPLAND et al., 2010), les hydrocarbones aromatiques polycycliques ou HAP (GSCHWEND \& HITES, 1981 ; SIMPSON et al., 1996 ; ROMANO et al., 2004 ; ANA, 2011), les polychlorobiphénils ou PCB (PAVLOU et al., 1982 ; FAVA et al., 2003 ; ROMANO et al., 2004 ; COLACICCO et al., 2010), et les composés de la famille du tributyl étain ou TBT (LANGSTON et al., 1987 ; BHOSLE et al., 2006 ; SAEKI et al., 2007 ; BLANCA, 2008). Les bio-essais qui ont été réalisés sur ces sédiments de dragage confirment le plus souvent leur écotoxicité (WONG et al., 1995 ; CLEMENT et al., 2009 ; MAMINDY-PAJANY et al., 2009 ; SRUT et al., 2010). Actuellement, il existe en France un stock important de sédiments contaminés (10 millions de tonnes environ), localisés dans les ports maritimes, qui ne peuvent pas être dragués en raison d'une évolution récente de la réglementation interdisant leur rejet en mer (IFREMER, 2001). Cela conduit à un nouveau gisement de matériaux pollués orphelins de filières, les filières classiques (incinération, mise en décharge,...) n'étant pas adaptées à leur traitement, ni sur le plan économique, ni sur le plan des volumes traitables. Dans l'objectif de trouver des solutions viables pour ces matériaux, des travaux de recherche et développement ont été initiés, notamment en France (GROSDEMANGE et al., 2008). Parmi les solutions de substitution envisagées, le remblaiement de carrières sèches représente une des filières les plus prometteuses, car une des plus adaptées sur le plan technique et économique, mais aussi parce qu'elle offre des capacités suffisantes pour gérer des volumes aussi importants. Cette filière nécessitait toutefois d'engager un programme de recherche d'envergure visant à proposer une "méthodologie d’évaluation des risques écologiques spécifique" permettant de valider, lors de chaque opération de remblaiement d'envergure, sa compatibilité avec les écosystèmes (terrestres et aquatiques) continentaux voisins, ainsi qu'avec la préservation de la ressource en eau (nappe phréatique).

Le but opérationnel de ce projet était donc l'élaboration d'une méthodologie d'Evaluation Des Risques Ecologiques (EDRE), adaptée à ces matériaux et à cette filière, ceci sur la base d'une recherche "amont" portant sur l'ensemble des facettes de la problématique (SEDIGEST, 2011).

\section{Matériels et méthodes}

D'une manière générale, sur le plan international, les méthodologies d'EDRE, développées pour divers scénarios de stockage ou d'usage de matériaux potentiellement polluants (SUTER, 1993 ; RIVIERE, 1998 ; US EPA, 1998 ; PERRODIN et al., 2000 ; 
Approche méthodologique pour la validation environnementale d'un projet de remblaiement de cavités terrestres de la zone littorale à l'aide de sédiments de dragage de ports maritimes traités : 2.3

CETMEF, 2001 ; ADEME, 2002 ; ECB, 2003 ; EMMANUEL et al., 2005 ; HAYET, 2006 ; RECORD, 2006), comprennent les quatre étapes suivantes :

a) formulation du problème,

b) caractérisation de l'exposition des écosystèmes cibles,

c) caractérisation des effets des flux polluants sur les écosystèmes,

d) caractérisation finale des risques écologiques.

Ces quatre étapes ont été élaborées dans le cadre du programme SEDIGEST (voir figure 1) pour un scénario de type "stockage en carrière de sédiments pollués préalablement traités".

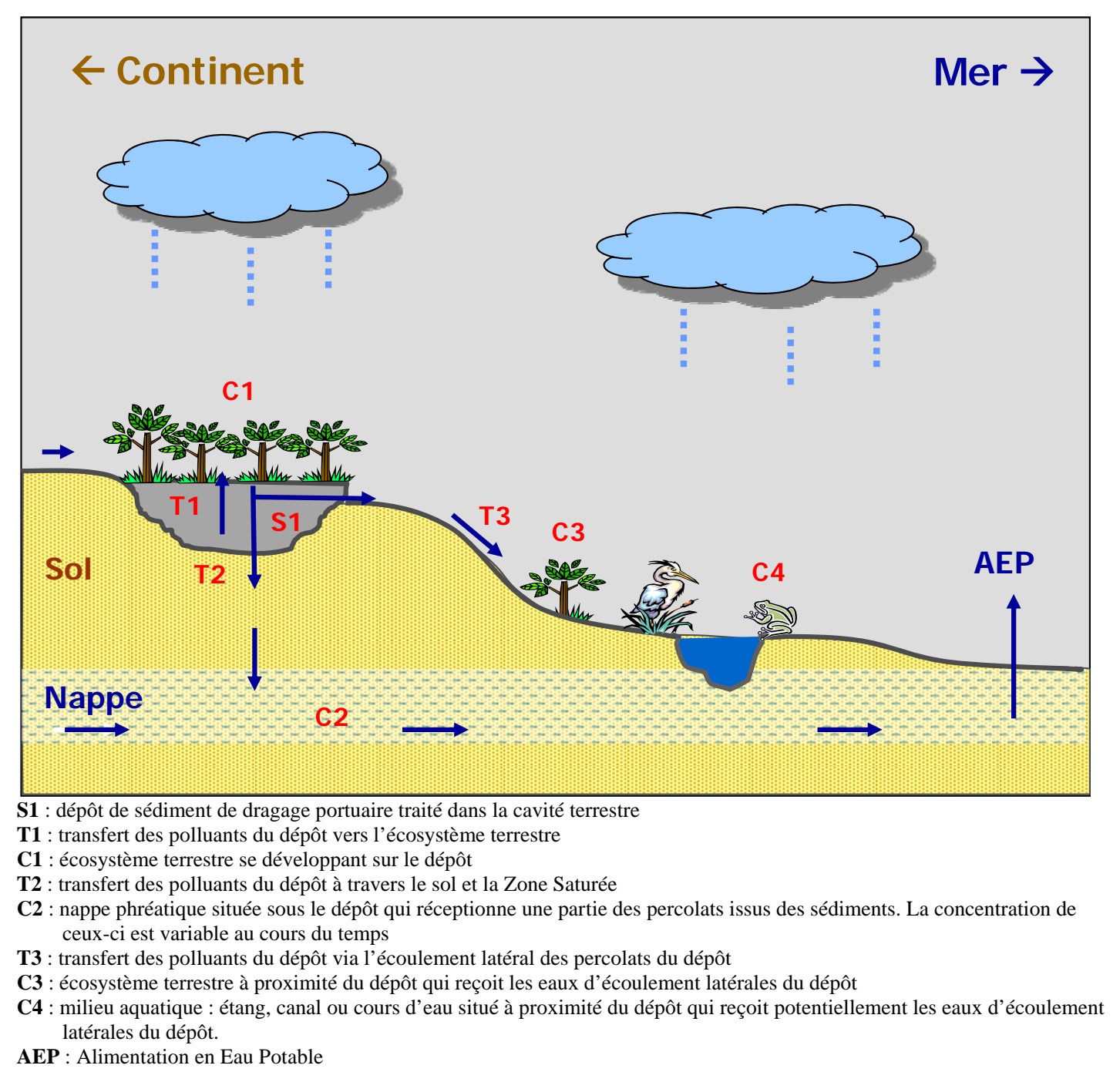

Figure 1. Scénario élaboré.

Les principaux blocages scientifiques qui s'opposaient à la rédaction d'une telle méthodologie étaient pour l'essentiel liés à la compréhension des différents mécanismes physiques, chimiques et biologiques qui interviennent lors du stockage à terre de tels 
matériaux salins pollués, notamment lors de la phase de mise en dépôt, puis sous l'action de la pluie, et enfin lors du contact des eaux potentiellement contaminées qui en résultent avec les écosystèmes continentaux voisins. Ils concernent en particulier la nécessaire prise en compte de la problématique des changements d'échelles spatiales et temporelles en vue d'une prévision réaliste des émissions de polluants et de leurs (non) impacts à long terme sur l'environnement.

Trois matrices sédimentaires (deux du Var et une du Finistère) ont été sélectionnées pour le programme sur la base d'un compromis entre le niveau de contamination et le type de prétraitement concerné. Trois pilotes lysimétriques ont ensuite été mis en œuvre en prenant en considération les résultats obtenus par des essais hydromécaniques sur chacun des trois sédiments. Ils ont permis de simuler les conditions de stockage, avec réalisation du bilan hydrique sur une durée de douze mois, et de collecter des percolats régulièrement analysés et utilisés pour les essais d’écotoxicité destinés à évaluer les impacts sur les écosystèmes voisins du dépôt. D'autre part, suite à l'établissement d'une typologie des cavités terrestres présentes dans les deux départements pilotes de SEDIGEST (Var et Finistère), deux sites expérimentaux ont été retenus pour les investigations de terrain. Sur ces sites, une analyse structurale, une prospection radar et sismique réfraction ont été effectuées sur trois zones dans une carrière du Var et sur deux zones dans une carrière du Finistère.

\section{Résultats}

\subsection{Modèle conceptuel global}

Pour visualiser l'ensemble des interactions entre les sources identifiées et les populations cibles, un modèle conceptuel a été élaboré (voir figure 2), précisant les sources d'émission, les voies de transfert et les écosystèmes cibles, ainsi que les différents éléments quantitatifs ou qualitatifs caractéristiques du scénario (ratios massiques/volumiques, facteurs de dilution, ou organismes et paramètres d'effet à tester). Les résultats de l'analyse des caractéristiques hydromécaniques du dépôt à terre ont permis par ailleurs de préciser les conditions de mise en œuvre optimum des trois matrices sédimentaires lors du remblaiement de carrières.

\subsection{Emissions du dépôt}

La caractérisation des émissions potentielles de polluants a été réalisée, via une procédure basée sur l'utilisation d'une batterie de tests de comportement complémentaires visant à évaluer la mobilisation potentielle des polluants présents dans des matrices sédimentaires portuaires traitées ou non. Les résultats montrent :

a) un faible risque de mobilisation des métaux cibles ( $\mathrm{As}, \mathrm{Cu}, \mathrm{Pb}$ et $\mathrm{Zn}$ ) au $\mathrm{pH}$ naturel mais une instabilité de ce dernier ; 
Approche méthodologique pour la validation environnementale d'un projet de remblaiement de cavités terrestres de la zone littorale à l'aide de sédiments de dragage de ports maritimes traités : 2.5

b) un risque à plus ou moins long terme dans des conditions de lixiviation particulières (milieu complexant, milieu acide,...) et une mise en évidence des effets des contraintes environnementales (carbonatation, eaux de pluie, anaérobiose,...) ;

c) un rôle prépondérant des carbonates sur le contrôle du $\mathrm{pH}$ et la mobilisation des polluants ;

d) une réactivité des sulfures (pyrite) ;

e) un rôle prépondérant de la matière organique: solubilisation et minéralisation observées en conditions de $\mathrm{pH}$ basiques ou fortement acides ;

f) une forte contamination en Hydrocarbures Aromatiques Polycycliques (HAPs).

La modélisation géochimique de l'émission des polluants a ensuite permis de mettre en évidence la cohérence et la complémentarité des trois approches menées (physicochimie, lixiviation et modélisation). La modélisation des émissions apparait comme un outil de compréhension intéressant vis-à-vis de la connaissance des sédiments, et elle peut être utilisée comme un outil d'aide à la décision permettant d'encadrer les conditions possibles du stockage.

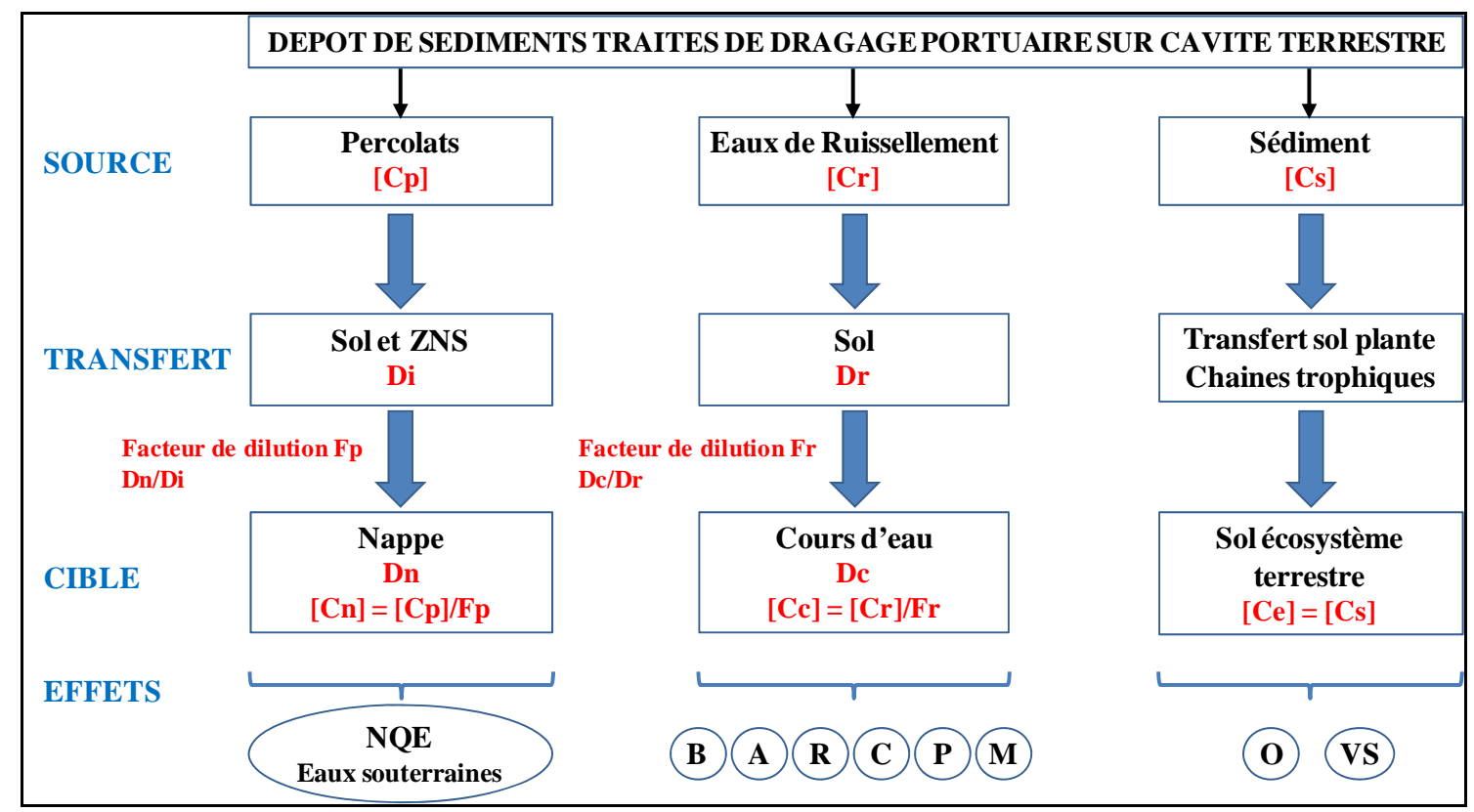

Cp : concentration du percolat, $\mathrm{Cr}$ : concentration dans l'eau de ruissellement, Cs concentration dans le sédiment, ZNS : Zone Non Saturée, Ce : concentration d'exposition, Dn Dilution dans la nappe, Dc dilution dans le cours d'eau, B : Bactéries, A : Algues, R : Rotifères, C : Crustacés, P : Poissons, M : Micro-organismes

Figure 2. Modèle conceptuel élaboré.

\subsection{Transfert des polluants}

Suite aux analyses structurales sur les deux sites pilotes, des modèles d'écoulement 3D ont permis l'étude des transferts d'eau et de polluants afin d'évaluer l'impact de sédiments potentiellement pollués sur le milieu souterrain. 


\subsection{Impact des polluants}

L’impact sur les écosystèmes terrestres situés à la surface du dépôt, abordée via l'évaluation des effets sur la germination et la croissance de plantes (Lolium perenne et une plante halophyte Armeria maritima), a montré que la germination et de développement des plantules étaient possibles pour certains sédiments après plusieurs années de lessivage de la surface du dépôt par les eaux météorites.

Concernant l'étude de l'impact sur les milieux aquatiques périphériques, un diagnostic fonctionnel des milieux aquatiques présents dans les bassins de drainage des deux sites pilote a tout d'abord été réalisé pour déterminer la valeur patrimoniale et fonctionnelle des écosystèmes aquatiques et estimer la vulnérabilité de ces milieux aux effluents issus des dépôts. Les principaux enseignements tirés de cette étude résident dans le fait qu'un des risques identifiés pour les communautés aquatiques est l'eutrophisation qui peut être provoquée par la libération de phosphates initialement présents dans les sédiments du cours d'eau, sous l'action d'une réaction en cascade initiée par les sulfates apportés par les percolats des sédiments en dépôt (SMOLDERS et al., 2006). Cette étude a ensuite été complétée à l'aide de tests écotoxicologiques monospécifiques normalisés [NF EN ISO 6341 (T90-301), 1996 ; ISO 11348-3, 1998 ; ISO 20079, 2005 ; NF EN ISO 8662 (T90-304), 2005 ; PR NF ISO 20666, 2007)]. Il ressort de cette étude : (i) que la bactérie $V$. fisheri n'est pas sensible aux percolats de certains sédiments, que le rotifère et l'algue verte sont les organismes les plus sensibles de la batterie et que la toxicité n'évolue pas significativement dans le temps ; (ii) qu’un sédiment chaulé est très toxique pour les organismes aquatiques.

Les essais sur lignées cellulaires de poisson ne montrent aucune génotoxicité primaire mais cela reste à vérifier en fonction du temps d'exposition.

Des essais en microcosmes aquatiques de 2 litres ont fourni des résultats cohérents avec ceux obtenus avec les essais mono-spécifiques, ce qui a permis de consolider les résultats.

\subsection{Evaluation des risques écologiques}

A l'issue de l'ensemble des travaux précédents, et de la prise en compte des données et contraintes techniques et économiques du terrain, une proposition méthodologique a été élaborée avec quatre niveaux de complexité possibles :

a) la première, dénommée "approche substances" (voir figure 3), consiste à comparer le niveau de concentration des différents polluants prévus dans les milieux cibles (PECmg/l) (via la réalisation d'un essai de percolation en lysimètre de laboratoire sur les sédiments étudiés, suivi de la modélisation des transferts dans les sols et la nappe phréatique), avec les concentrations maximum acceptables par les écosystèmes concernés accessibles dans les bases de données internationales (PNECmg/l); 
Approche méthodologique pour la validation environnementale d'un projet de remblaiement de cavités terrestres de la zone littorale à l'aide de sédiments de dragage de ports maritimes traités : 2.7

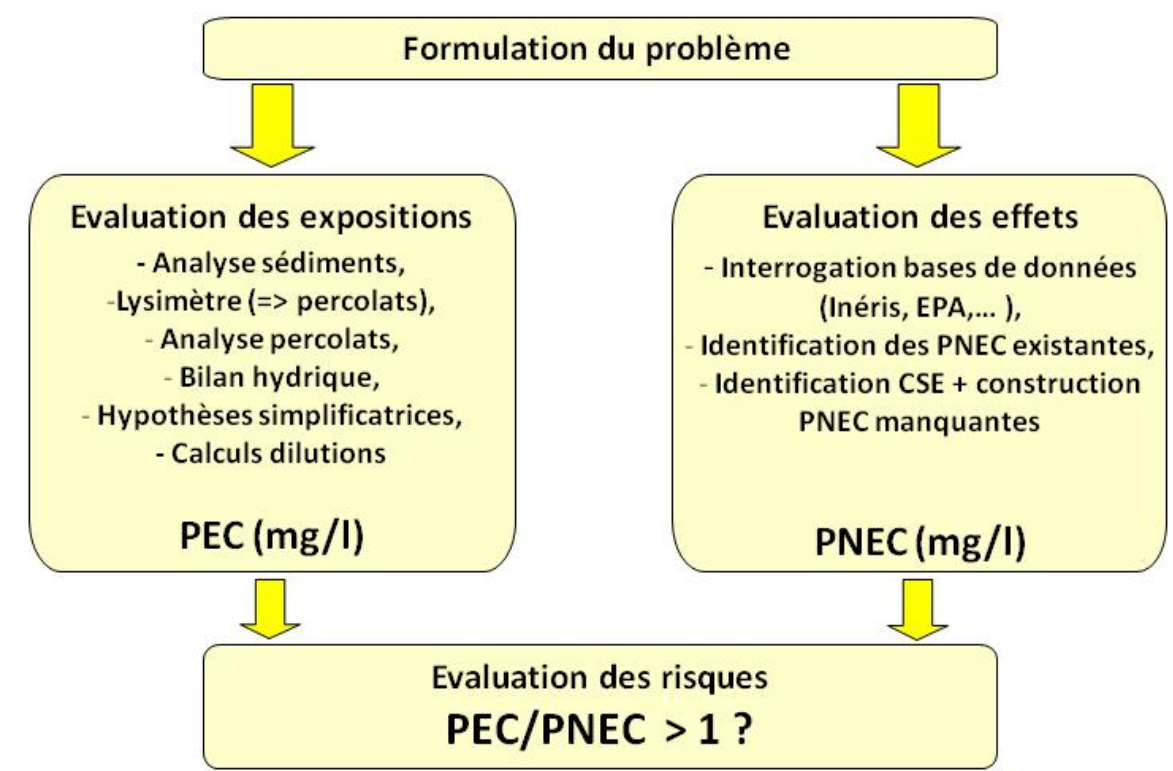

Figure 3. Approche "Substances".

b) la seconde, dénommée "approche matrice" (voir figure 4), consiste à comparer le pourcentage de percolat prévu dans les milieux récepteurs cibles (PEC\%) avec le pourcentage de percolat admissible dans chacun de ces milieux (PNEC\%), via la réalisation d'essais d'écotoxicité monospécifiques sur ce percolat ;

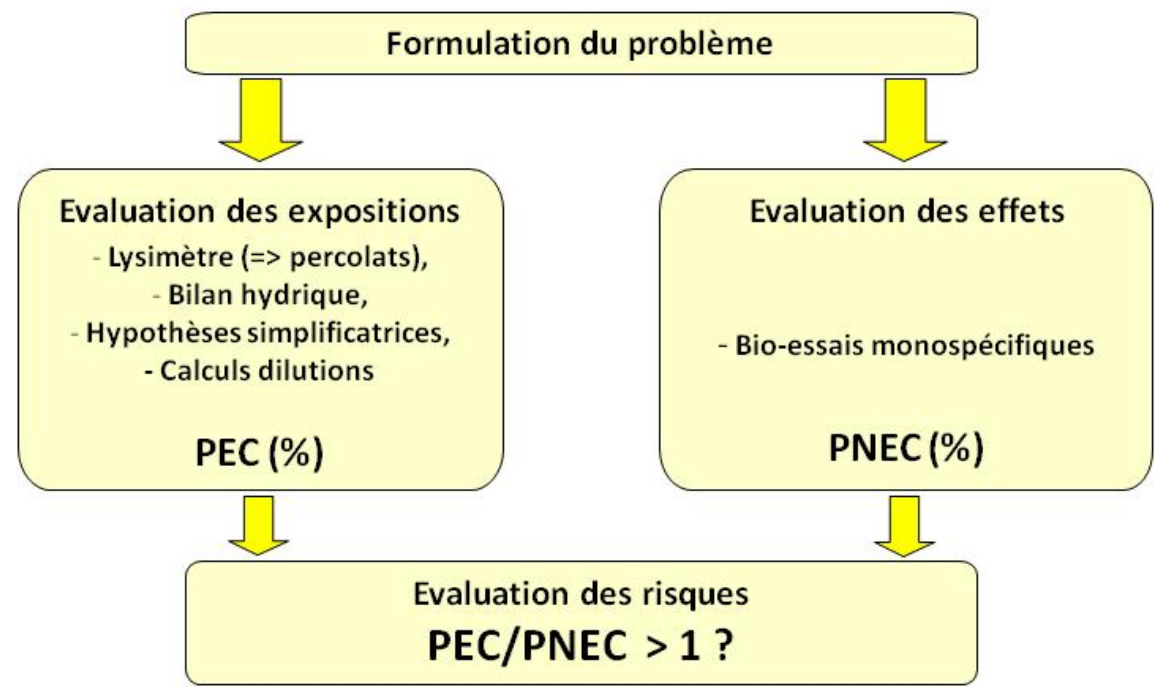

Figure 4. Approche "Matrice".

c) la troisième, dénommée "approche substances approfondie" (voir figure 5), correspond à l'approche substance ci-dessus, consolidée par une série d'essais de comportement des sédiments à des variations de conditions environnementales $(\mathrm{pH}$, potentiel REDOX, ...) de manière à vérifier la validité de la prédiction effectuée sur le long terme ; 


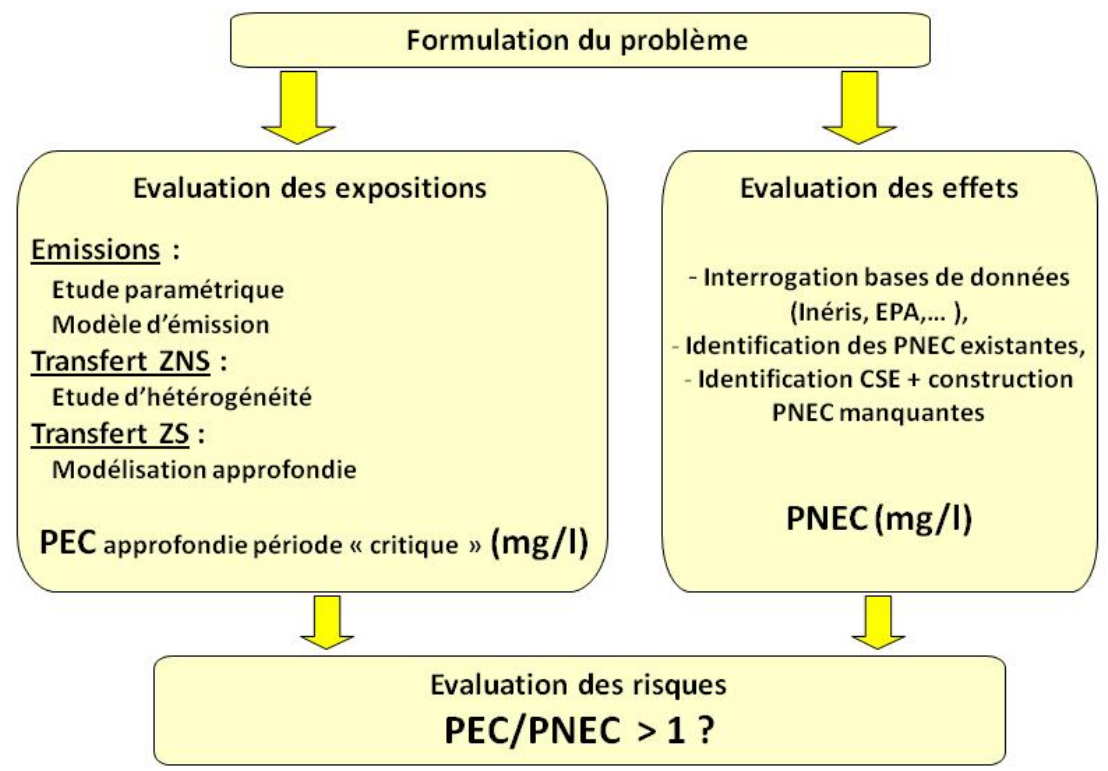

Figure 5. Approche "Substances" approfondie.

d) la quatrième, dénommée "approche matrice approfondie" (voir figure 6), consiste à compléter l'évaluation des effets du percolat présentée ci-dessus par la mise en œuvre d'essais en microcosmes sur ces mêmes percolats.

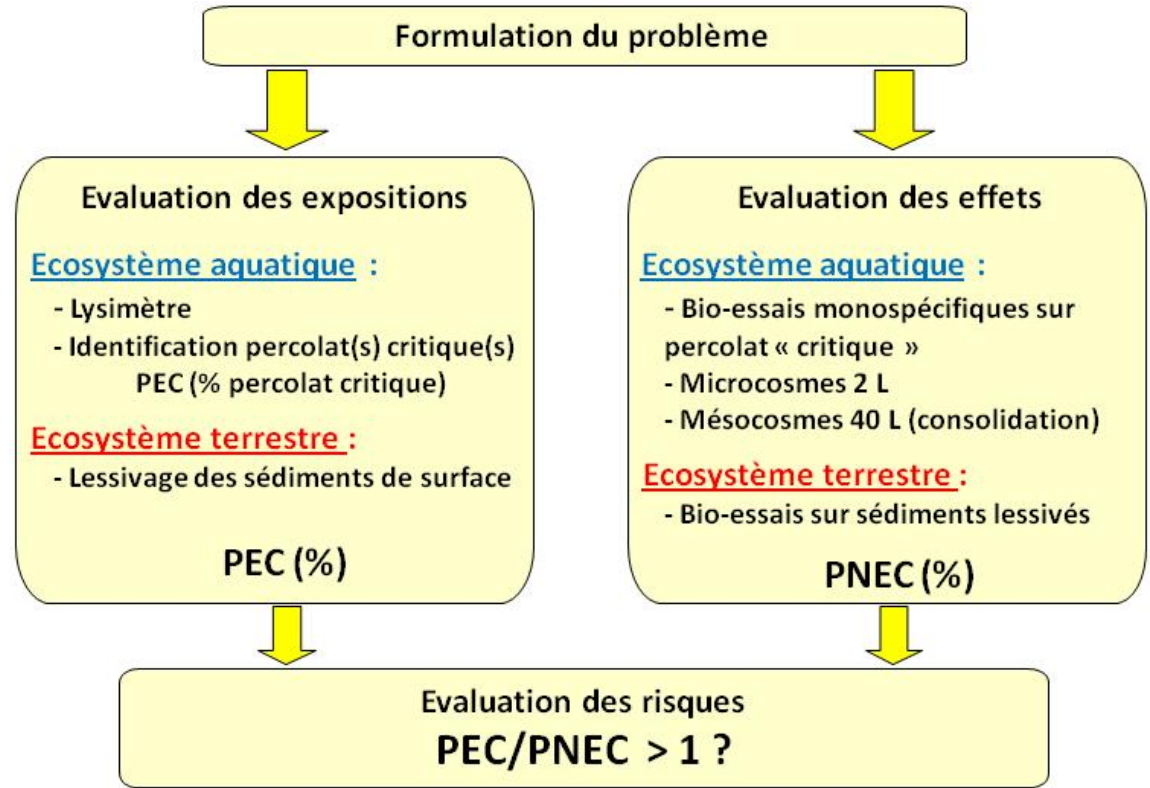

Figure 6. Approche "Matrice" approfondie

La mise à disposition de ces quatre méthodologies permet aux gestionnaires de mobiliser progressivement, et si nécessaire, les approches les plus complexes, et les plus coûteuses, en fonction des enjeux locaux et des résultats des approches précédentes. 
Approche méthodologique pour la validation environnementale d'un projet de remblaiement de cavités terrestres de la zone littorale à l'aide de sédiments de dragage de ports maritimes traités : 2.9

\section{Conclusions}

L'ensemble des résultats expérimentaux et théoriques acquis au cours du programme SEDIGEST ont permis de formuler, et de valider à l'échelle pilote, une approche méthodologique multi-niveaux, adaptée pour la validation environnementale d'un projet de remblaiement de cavités terrestres de la zone littorale à l'aide de sédiments de dragage d'un port maritime. Il reste maintenant à appliquer cette méthodologie sur un certain nombre de sites de dépôt pilotes, qu'il conviendra d'instrumenter et de suivre pendant plusieurs années afin de préciser et de valider la méthodologie à grande échelle. L'élargissement du domaine d'application de la méthodologie à d'autres filières de gestion des sédiments de dragage de ports maritimes est envisageable, à condition toutefois de réaliser préalablement des travaux du même type que ceux du programme SEDIGEST, travaux qui seront définis précisément à l'issue de la première étape de l'EDRE, c'est à dire la "formulation du problème".

\section{Remerciements}

Nous remercions l'ensemble des équipes ayant participé au programme de recherche SEDIGEST, appartenant aux organismes suivants (détail des intervenants sur www.sedigest.org) : ENTPE, INSA de Lyon, BRGM, INSAVALOR, INERIS, IN VIVO Environnement, CETMEF, CG 83 et CG 29.

Nous remercions également l'Agence Nationale de la Recherche et les Pôles de Compétitivité AXELERA et Mer PACA et Mer Bretagne pour respectivement l'aide financière et la labellisation attribuées au projet SEDIGEST.

\section{Références bibliographiques}

ADEME (2002). Évaluation de l'écocompatibilité des scénarios de stockage et de valorisation des déchets. Agence De l'Environnement et de la Maîtrise de l'Énergie, Angers, $148 \mathrm{p}$.

ANA A.-J. (2011). Polycyclic aromatic hydrocarbons in marine sediments from the Rijeka Bay area, Northern Adriatic, Croatia, 1998-2006. Marine Pollution Bulletin 62, pp 863-869.

ANDERSEN H.V., KJÃ,LHOLT J., POLL C., DAHL S.Ã.G., STUER-LAURIDSEN F., PEDERSEN F., BJÃ,RNESTAD E. (1998). Environmental risk assessment of surface water and sediments in Copenhagen harbour. Water Science and Technology 37, pp 263-272.

BHOSLE N.B., GARG A., HARJI R., JADHAV S., SAWANT S.S., KRISHNAMURTHY V., ANIL C. (2006). Butyltins in the sediments of Kochi and Mumbai harbours, west coast of India. Environment International 32, pp 252-258.

BLANCA A.-L. (2008). Environmental levels, toxicity and human exposure to tributyltin (TBT)-contaminated marine environment. A review. Environment International 34, pp 292-308. 
CETMEF (2001). Évaluation écotoxicologique de sédiments contaminés ou de matériaux de dragage. Direction de la Recherche et des Affaires Scientifiques et Techniques du Ministère de l'Equipement des Transports et du Logement et par Voies navigables de France, Centre d'Etudes Techniques Maritimes et Fluviales, 12 p.

CLEMENT B. (2010). Ecotoxicological risk assessment of a scenario of terrestrial quarries filled with sea harbor contaminated sediments using laboratory microcosm assays. IInd International Symposium on sediment management, 11-13 may 2010, Casablanca, Maroc.

COLACICCO A., DE GIOANNIS G., MUNTONI A., PETTINAO E., POLETTINI A., POMI, R. (2010). Enhanced electrokinetic treatment of marine sediments contaminated by heavy metals and PAHs. Chemosphere 81, pp 46-56.

ECB (2003). Technical Guidance Document (TGD) in support of Commission Directive 93/67/EEC on Risk Assessment for new notified substances, Commission Regulation (EC) No 1488/94 on Risk Assessment for existing substances and Directive 98/8/EC of the European Parliament and of the Council concerning the placing of biocidal products on the market. European Chemical Bureau, Ispra (Italy), 1044 p.

EMMANUEL E., PERRODIN Y., KECK G., BLANCHARD J.M., VERMANDE P. (2005). Ecotoxicological risk assessment of hospital wastewater: a proposed framework for raw effluents discharging into urban sewer network. J. Hazard. Mater. 117, pp 1-11. FAVA F., GENTILUCCI S., ZANAROLI G. (2003). Anaerobic biodegradation of weathered polychlorinated biphenyls (PCBs) in contaminated sediments of Porto Marghera (Venice Lagoon, Italy). Chemosphere 53, pp 101-109.

GROSDEMANGE D., LEVEQUE F., DROUSIE D., AQUA J.L., MEHU J., BAZIN C. (2008). The SEDIMARD project: presentation and results. International Symposium on Sédiment Management I2SM, Lille, pp. 181-186.

GSCHWEND P.M., HITES R.A. (1981). Fluxes of polycyclic aromatic hydrocarbons to marine and lacustrine sediments in the northeastern United States. Geochimica et Cosmochimica Acta 45, pp 2359-2367.

HAYET A. (2006). Variabilité des méthodologies d'évaluation des risques écologiques: conséquences et perspectives d'améliorations. Mémoire de master recherche en ingénierie de la santé. Mention Santé - Environnement. Institut Lillois d'Ingénierie de la Santé, Université de Lille 2, 95 p.

IFREMER (2001). Dredging and marine environment. $20 \mathrm{p}$. http://envlit.ifremer.fr/var/envlit/storage/documents/dossiers/bioevaluation/site/index.htm

ISO 11348-3 (1998). Qualité de l'eau - Détermination de l'effet inhibiteur d'échantillons d'eau sur la luminescence de Vibrio fischeri (Essai de bactéries luminescentes). Partie 3 : méthode utilisant des bactéries lyophilisées. Norme internationale.

ISO 20079 (2005). Qualité de l'eau - Détermination de l'effet toxique des constituants de l'eau et des eaux résiduaires vis-à-vis des lentilles d'eau (Lemna minor) - Essai d'inhibition de la croissance des lentilles d'eau. Norme internationale. 
Approche méthodologique pour la validation environnementale d'un projet de remblaiement de cavités terrestres de la zone littorale à l'aide de sédiments de dragage de ports maritimes traités : 2.11

LANGSTON W.J., BURT G.R., MINGJIANG Z. (1987). Tin and organotin in water, sediments, and benthic organisms of Poole Harbour. Marine Pollution Bulletin 18, pp 634-639.

LAU M.M.-M., ROOTHAM R.C., BRADLEY G.C. (1993). A Strategy for the Management of Contaminated Dredged Sediment in Hong Kong. Journal of Environmental Management 38, pp 99-114.

LEPLAND A., ANDERSEN T.R.J., LEPLAND A., ARP H.P.H., ALVE E., BREEDVELD G.D., RINDBY A. (2010). Sedimentation and chronology of heavy metal pollution in Oslo harbor, Norway. Marine Pollution Bulletin 60, pp 1512-1522. MAMINDY-PAJANY Y., LIBRALATO G., ROMÉO M., HUREL C., LOSSO C., GHIRARDINI A.V., MARMIER N. (2009). Ecotoxicological evaluation of Mediterranean dredged sediment ports based on elutriates with oyster embryotoxicity tests after composting process. Water Research 44, pp 1986-1994.

MEEDDAT (2008). Circulaire du 04/07/08 relative à la procédure concernant la gestion des sédiments lors de travaux ou d'opérations impliquant des dragages ou curages maritimes et fluviaux. BO du MEEDDAT n 2008/15 (France).

NF EN ISO 6341 (T90-301) (1996). Qualité de l'eau : Détermination de l'inhibition de la mobilité de Daphnia magna Straus (Cladocera, Crustacea) - Essai de toxicité aiguë.

NF EN ISO 8662 (T90-304) (2005). Qualité de l'eau - Essai d'inhibition de la croissance des algues d'eau douce avec des algues vertes unicellulaires.

PAVLOU S.P., HOM W., DEXTER R.N., ANDERSON D.E., QUINLAN E.A. (1982). Release, distribution, and impacts of polychlorinated biphenyls (PCB) induced by dredged material disposal activities at a deep-water estuarine site. Environment International 7, pp 99-117.

PERRODIN Y., GRELIER-VOLATIER L., BARNA R., GOBBEY A. (2000). Assessment of the ecocompatibility of waste disposal or waste use scenarios: towards the elaboration and implementation of a comprehensive methodology. Waste Management Series 1, pp 504-512.

PR NF ISO 20666 (2007). Qualité de l'eau - Détermination de la toxicité chronique visà-vis de Brachionus calyciflorus en $48 \mathrm{~h}$.

RECORD (2006). Évaluation et acceptabilité des risques environnementaux. Méthodes d'évaluation, analyse comparative. Etude sociologique des représentations des risques et synthèse bibliographique. 227 p.

RIVIERE J.L. (1998). Évaluation du risque écologique des sols pollués, Paris.

ROMANO E., AUSILI A., ZHAROVA N., CELIA MAGNO M., PAVONI B., GABELLINI M. (2004). Marine sediment contamination of an industrial site at Port of Bagnoli, Gulf of Naples, Southern Italy. Marine Pollution Bulletin 49, pp 487-495.

SAEKI K., NABESHIMA A., KUNITO T., OSHIMA Y. (2007). The stability of butyltin compounds in a dredged heavily-contaminated sediment. Chemosphere 68, pp 1114-1119. 
SEDIGEST (2011). Programme de recherche ANR SEDIGEST. http://www.sedigest.org/. In: ENTPE (Ed.), Vaulx en Velin.

SIMPSON C.D., MOSI A.A., CULLEN W.R., REIMER K.J. (1996). Composition and distribution of polycyclic aromatic hydrocarbon contamination in surficial marine sediments from Kitimat Harbor, Canada. Science of The Total Environment 181, pp 265-278.

SMOLDERS A.J.P., LAMERS L.P.M., LUCASSEN E.C.H.E.T., VAN DER VELDE G., ROELOFS J.G.M. (2006). Internal eutrophication: How it works and what to do about it: a review. Chemistry and Ecology 22, pp 93-111.

SRUT M., TRAVEN L., STAMBUK A., KRALJ S., ZAJA R., MICOVIC V., KLOBUCAR G.I.V. (2010). Genotoxicity of marine sediments in the fish hepatoma cell line PLHC-1 as assessed by the Comet assay. Toxicology in Vitro 25, pp 308-314.

SUTER G.W. (1993). Environmental Risk Assessment. Lewis Publishers, Chelsea.

US EPA (1998). Guidelines for Ecological Risk Assessment. United States Environmental Protection Agency, Washington, 188 p.

WONG Y.S., TAM N.F.Y., LAU P.S., XUE X.Z. (1995). The toxicity of marine sediments in Victoria Harbour, Hong Kong. Marine Pollution Bulletin 31, pp 464-470. 